\title{
Type Ia Supernovae: Progenitors and Evolution with Redshift
}

\author{
Ken'ichi Nomoto ${ }^{1}$, Hideyuki Umeda ${ }^{1}$, Chiaki Kobayashi ${ }^{1}$, \\ Izumi Hachisu $^{2}$, Mariko Kato ${ }^{3}$, \& Takuji Tsujimoto ${ }^{4}$ \\ ${ }^{1}$ Department of Astronomy and Research Center for the Early Universe, University of Tokyo \\ ${ }^{2}$ Department of Earth Science and Astronomy, College of Arts and Sciences, University of Tokyo \\ ${ }^{3}$ Department of Astronomy, Keio University, Hiyoshi, Yokohama, Japan \\ ${ }^{4}$ National Astronomical Observatory, Mitaka, Japan
}

To be published in the Proceedings of the 10th Annual October Astrophysics Conference in Maryland (11 - 13 October 1999) "COSMIC EXPLOSIONS!", ed. S.S. Holt and W.W. Zhang (American Institute of Physics)

\begin{abstract}
.
Relatively uniform light curves and spectral evolution of Type Ia supernovae (SNe Ia) have led to the use of SNe Ia as a "standard candle" to determine cosmological parameters. Whether a statistically significant value of the cosmological constant can be obtained depends on whether the peak luminosities of SNe Ia are sufficiently free from the effects of cosmic and galactic evolutions.

Here we first review the single degenerate scenario for the Chandrasekhar mass white dwarf (WD) models of SNe Ia. We identify the progenitor's evolution and population with two channels: (1) the WD+RG (red-giant) and (2) the WD+MS (near mainsequence He-rich star) channels. In these channels, the strong wind from accreting WDs plays a key role, which yields important age and metallicity effects on the evolution.

We then address the questions whether the nature of SNe Ia depends systematically on environmental properties such as metallicity and age of the progenitor system and whether significant evolutionary effects exist. We suggest that the variation of the carbon mass fraction $X(\mathrm{C})$ in the $\mathrm{C}+\mathrm{O}$ WD (or the variation of the initial WD mass) causes the diversity of the brightness of SNe Ia. This model can explain the observed dependences of SNe Ia brightness on the galaxy types and the distance from the galactic center.

Finally, applying the metallicity effect on the evolution of SN Ia progenitors, we make a prediction of the cosmic supernova rate history as a composite of the supernova rates in different types of galaxies.
\end{abstract}

\section{INTRODUCTION}

Type Ia supernovae (SNe Ia) are good distance indicators, and provide a promising tool for determining cosmological parameters (e.g., [1]). SNe Ia have been discovered up to $z \sim 1.32$ [2]. Both the Supernova Cosmology Project [3,4] and the High-z Supernova Search Team $[5,6]$ have suggested a statistically significant value for the cosmological constant. 
However, SNe Ia are not perfect standard candles, but show some intrinsic variations in brightness. When determining the absolute peak luminosity of high-redshift SNe Ia, therefore, these analyses have taken advantage of the empirical relation existing between the peak brightness and the light curve shape (LCS). Since this relation has been obtained from nearby SNe Ia only [7-9], it is important to examine whether it depends systematically on environmental properties such as metallicity and age of the progenitor system.

High-redshift supernovae present us very useful information, not only to determine cosmological parameters but also to put constraints on the star formation history in the universe. They have given the SN Ia rate at $z \sim 0.5$ [10] but will provide the SN Ia rate history over $0<z<1$. With the Next Generation Space Telescope, both SNe Ia and SNe II will be observed through $z \sim 4$. It is useful to provide a prediction of cosmic supernova rates to constrain the age and metallicity effects of the SN Ia progenitors.

SNe Ia have been widely believed to be a thermonuclear explosion of a massaccreting white dwarf (WD) (e.g., [11] for a review). However, the immediate progenitor binary systems have not been clearly identified yet [12]. In order to address the above questions regarding the nature of high-redshift SNe Ia, we need to identify the progenitors systems and examine the "evolutionary" effects (or environmental effects) on those systems.

In $\S 2$, we summarize the progenitors' evolution where the strong wind from accreting WDs plays a key role [13-15]. In $\S 3$, we address the issue of whether a difference in the environmental properties is at the basis of the observed range of peak brightness [17]. In $\S 4$, we make a prediction of the cosmic supernova rate history as a composite of the different types of galaxies [18].

\section{EVOLUTION OF PROGENITOR SYSTEMS}

There exist two models proposed as progenitors of SNe Ia: 1) the Chandrasekhar mass model, in which a mass-accreting carbon-oxygen $(\mathrm{C}+\mathrm{O}) \mathrm{WD}$ grows in mass up to the critical mass $M_{\text {Ia }} \simeq 1.37-1.38 M_{\odot}$ near the Chandrasekhar mass and explodes as an SN Ia (e.g., [19,20]), and 2) the sub-Chandrasekhar mass model, in which an accreted layer of helium atop a $\mathrm{C}+\mathrm{O}$ WD ignites off-center for a WD mass well below the Chandrasekhar mass (e.g., [21]). The early time spectra of the majority of SNe Ia are in excellent agreement with the synthetic spectra of the Chandrasekhar mass models, while the spectra of the sub-Chandrasekhar mass models are too blue to be comparable with the observations [22,23].

For the evolution of accreting WDs toward the Chandrasekhar mass, two sce-

narios have been proposed: 1) a double degenerate (DD) scenario, i.e., merging of double $\mathrm{C}+\mathrm{O}$ WDs with a combined mass surpassing the Chandrasekhar mass limit [24,25], and 2) a single degenerate (SD) scenario, i.e., accretion of hydrogen-rich matter via mass transfer from a binary companion (e.g., [26,20]). The issue of DD vs. SD is still debated (e.g., [12]), although theoretical modeling has indicated 
that the merging of WDs leads to the accretion-induced collapse rather than SN Ia explosion [27-29].

In the SD Chandrasekhar mass model for SNe Ia, a WD explodes as a SN Ia only when its rate of the mass accretion $(\dot{M})$ is in a certain narrow range (e.g., $[26,30])$. In particular, if $\dot{M}$ exceeds the critical rate $\dot{M}_{\mathrm{b}}$, the accreted matter extends to form a common envelope [31]. This difficulty has been overcome by the WD wind model (see below). For the actual binary systems which grow the WD mass $\left(M_{\mathrm{WD}}\right)$ to $M_{\text {Ia }}$, the following two systems are appropriate. One is a system consisting of a mass-accreting WD and a lobe-filling, more massive, slightly evolved main-sequence or sub-giant star (hereafter "WD+MS system"). The other system consists of a WD and a lobe-filling, less massive, red-giant (hereafter "WD+RG system").

\section{A White dwarf winds}

Optically thick WD winds are driven when the accretion rate $\dot{M}$ exceeds the critical rate $\dot{M}_{\mathrm{b}}$. Here $\dot{M}_{\mathrm{b}}$ is the rate at which steady burning can process the accreted hydrogen into helium as $\dot{M}_{\mathrm{b}} \approx 0.75 \times 10^{-6}\left(\frac{M_{\mathrm{wD}}}{M_{\odot}}-0.40\right) M_{\odot} \mathrm{yr}^{-1}$.

With such a rapid accretion, the WD envelope expands to $R_{\mathrm{ph}} \sim 0.1 R_{\odot}$ and the photospheric temperature decreases below $\log T_{\mathrm{ph}} \sim 5.5$. Around this temperature, the shoulder of the strong peak of OPAL Fe opacity [32] drives the radiationdriven wind $[13,15]$. The ratio of $v_{\mathrm{ph}} / v_{\mathrm{esc}}$ between the photospheric velocity and the escape velocity at the photosphere depends on the mass transfer rate and $M_{\mathrm{WD}}$. (see Fig.6 in [15]). We call the wind strong when $v_{\mathrm{ph}}>v_{\mathrm{esc}}$. When the wind is strong, $v_{\mathrm{ph}} \sim 1000 \mathrm{~km} \mathrm{~s}^{-1}$ being much faster than the orbital velocity.

If the wind is sufficiently strong, the WD can avoid the formation of a common

envelope and steady hydrogen burning increases its mass continuously at a rate $\dot{M}_{\mathrm{b}}$ by blowing the extra mass away in a wind. When the mass transfer rate decreases below this critical value, optically thick winds stop. If the mass transfer rate further decreases below $\sim 0.5 \dot{M}_{\mathrm{b}}$, hydrogen shell burning becomes unstable to trigger very weak shell flashes but still burns a large fraction of accreted hydrogen.

The steady hydrogen shell burning converts hydrogen into helium atop the $\mathrm{C}+\mathrm{O}$ core and increases the mass of the helium layer gradually. When its mass reaches a certain value, weak helium shell flashes occur. Then a part of the envelope mass is blown off but a large fraction of $\mathrm{He}$ can be burned to $\mathrm{C}+\mathrm{O}$ [33] to increase the WD mass. In this way, strong winds from the accreting WD play a key role to increase the WD mass to $M_{\text {Ia }}$.

\section{B WD+RG system}

This is a symbiotic binary system consisting of a WD and a low mass red-giant (RG). A full evolutionary path of the WD+RG system from the zero age mainsequence stage to the SN Ia explosion is described in $[15,16]$. The occurrence frequency of SNe Ia through this channel is much larger than the earlier scenario, because of the following two evolutionary processes, which have not considered before. 
(1) Because of the AGB wind, the WD + RG close binary can form from a wide binary even with such a large initial separation as $a_{i} \lesssim 40,000 R_{\odot}$. Our earlier estimate [13] is constrained by $a_{i} \lesssim 1,500 R_{\odot}$.

(2) When the RG fills its inner critical Roche lobe, the WD undergoes rapid mass accretion and blows a strong optically thick wind. Our earlier analysis has shown that the mass transfer is stabilized by this wind only when the mass ratio of RG/WD is smaller than 1.15. Our new finding is that the WD wind can strip mass from the RG envelope, which could be efficient enough to stabilize the mass transfer even if the RG/WD mass ratio exceeds 1.15. If this mass-stripping effect is strong enough, though its efficiency $\eta_{\text {eff }}$ is subject to uncertainties, the symbiotic channel can produce SNe Ia for a much (ten times or more) wider range of the binary parameters than our earlier estimation.

With the above two new effects (1) and (2), the WD+RG (symbiotic) channel can account for the inferred rate of SNe Ia in our Galaxy. The immediate progenitor binaries in this symbiotic channel to SNe Ia may be observed as symbiotic stars, luminous supersoft X-ray sources, or recurrent novae like T CrB or RS Oph, depending on the wind status.

\section{WD+MS system}

In this scenario, a $\mathrm{C}+\mathrm{O} \mathrm{WD}$ is originated, not from an $\mathrm{AGB}$ star with a $\mathrm{C}+\mathrm{O}$ core, but from a red-giant star with a helium core of $\sim 0.8-2.0 M_{\odot}$. The helium star, which is formed after the first common envelope evolution, evolves to form a $\mathrm{C}+\mathrm{O}$ WD of $\sim 0.8-1.1 M_{\odot}$ with transferring a part of the helium envelope onto the secondary main-sequence star. A full evolutionary path of the WD+MS system from the zero age main-sequence stage to the SN Ia explosion is described in [14].

This evolutionary path provides a much wider channel to SNe Ia than previous scenarios. A part of the progenitor systems are identified as the luminous supersoft X-ray sources [34] during steady H-burning (but without wind to avoid extinction), or the recurrent novae like U Sco if H-burning is weakly unstable. Actually these objects are characterized by the accretion of helium-rich matter.

\section{Realization frequency}

For an immediate progenitor system WD+RG of SNe Ia, we consider a close binary initially consisting of a $\mathrm{C}+\mathrm{O}$ WD with $M_{\mathrm{WD}, 0}=0.6-1.2 M_{\odot}$ and a lowmass red-giant star with $M_{\mathrm{RG}, 0}=0.7-3.0 M_{\odot}$ having a helium core of $M_{\mathrm{He}, 0}=$ $0.2-0.46 M_{\odot}$. The initial state of these immediate progenitors is specified by three parameters, i.e., $M_{\mathrm{WD}, 0}, M_{\mathrm{RG}, 0}=M_{\mathrm{d}, 0}$, and the initial orbital period $P_{0}\left(M_{\mathrm{He}, 0}\right.$ is determined if $P_{0}$ is given).

We follow binary evolutions of these systems and obtain the parameter range(s) which can produce an SN Ia. In Figure 1, the region enclosed by the thin solid line produces SNe Ia for several cases of the initial WD mass, $M_{\mathrm{WD}, 0}=0.75-1.1 M_{\odot}$. For smaller $M_{\mathrm{WD}, 0}$, the wind is weaker, so that the $\mathrm{SN}$ Ia region is smaller. The 


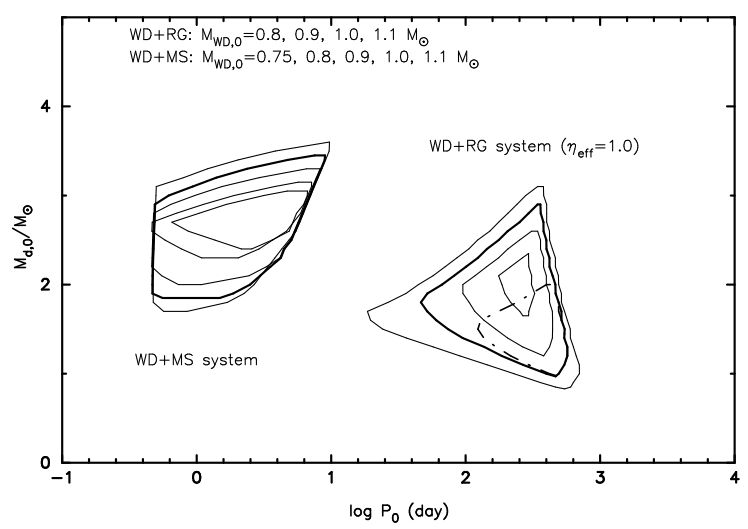

FIGURE 1. The region to produce SNe Ia in the $\log P_{0}-M_{\mathrm{d}, 0}$ plane for five initial WD masses of $0.75 M_{\odot}, 0.8 M_{\odot}, 0.9 M_{\odot}, 1.0 M_{\odot}$ (heavy solid line), and $1.1 M_{\odot}$. The region of $M_{\mathrm{WD}, 0}=0.7 M_{\odot}$ almost vanishes for both the WD+MS and WD+RG systems, and the region of $M_{\mathrm{WD}, 0}=0.75 M_{\odot}$ vanishes for the $\mathrm{WD}+\mathrm{RG}$ system. Here, we assume the stripping efficiency of $\eta_{\mathrm{eff}}=1$. For comparison, we show only the region of $M_{\mathrm{WD}, 0}=1.0 M_{\odot}$ for a much lower efficiency of $\eta_{\text {eff }}=0.3$ by a dash-dotted line.

regions of $M_{\mathrm{WD}, 0}=0.6 M_{\odot}$ and $0.7 M_{\odot}$ vanish for both the $\mathrm{WD}+\mathrm{MS}$ and $\mathrm{WD}+\mathrm{RG}$ systems.

In the outside of this region, the outcome of the evolution at the end of the calculations is not an SN Ia but one of the followings: (i) Formation of a common envelope for too large $M_{\mathrm{d}}$ or $P_{0} \sim$ day, where the mass transfer is unstable at the beginning of mass transfer. (ii) Novae or strong hydrogen shell flash for too small $M_{\mathrm{d}, 0}$, where the mass transfer rate becomes below $10^{-7} M_{\odot} \mathrm{yr}^{-1}$. (iii) Helium core flash of the red giant component for too long $P_{0}$, where a central helium core flash ignites, i.e., the helium core mass of the red-giant reaches $0.46 M_{\odot}$. (iv) Accretioninduced collapse for $M_{\mathrm{WD}, 0}>1.2 M_{\odot}$, where the central density of the WD reaches $\sim 10^{10} \mathrm{~g} \mathrm{~cm}^{-3}$ before heating wave from the hydrogen burning layer reaches the center. As a result, the WD undergoes collapse due to electron capture without exploding as an SN Ia [30].

It is clear that the new region of the WD+RG system is not limited by the condition of $q<1.15$, thus being ten times or more wider than the region of [13]'s model (depending on the the stripping efficiency of $\eta_{\text {eff }}$ ).

The WD+MS progenitor system can also be specified by three initial parameters: the initial $\mathrm{C}+\mathrm{O}$ WD mass $M_{\mathrm{WD}, 0}$, the mass donor's initial mass $M_{\mathrm{d}, 0}$, and the orbital period $P_{0}$. For $M_{\mathrm{WD}, 0}=1.0 M_{\odot}$, the region producing an SN Ia is bounded by $M_{\mathrm{d}, 0}=1.8-3.2 M_{\odot}$ and $P_{0}=0.5-5 \mathrm{~d}$ as shown by the solid line in Figure 1 . The upper and lower bounds are respectively determined by the common envelope formation (i) and nova-like explosions (ii) as above. The left and right bounds are determined by the minimum and maximum radii during the main sequence of the donor star [14].

We estimate the rate of SNe Ia originating from these channels in our Galaxy 


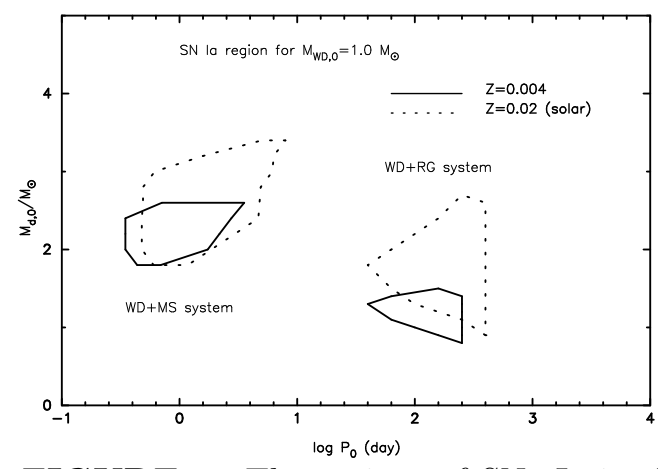

FIGURE 2. The regions of $\mathrm{SNe}$ Ia is plotted in the initial orbital period vs. the initial companion mass diagram for the initial WD mass of $M_{\mathrm{WD}, 0}=1.0 M_{\odot}$. The dashed and solid lines represent the cases of solar abundance $(Z=0.02)$ and much lower metallicity of $Z=0.004$, respectively. The left and the right regions correspond to the $\mathrm{WD}+\mathrm{MS}$ and the $\mathrm{WD}+\mathrm{RG}$ systems, respectively.

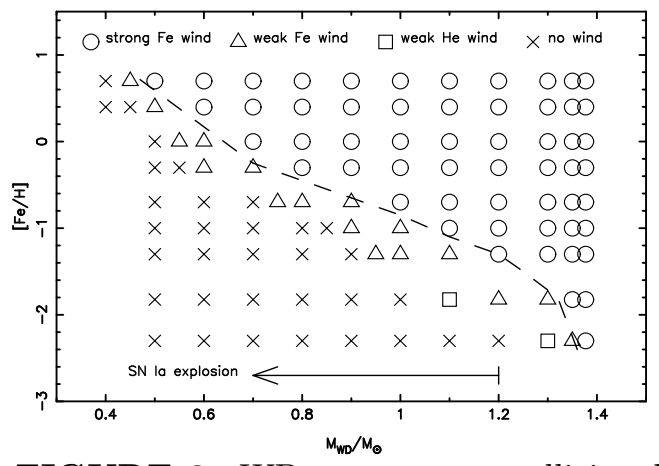

FIGURE 3. WD mass vs. metallicity diagram showing the metallicity dependence of optically thick winds. We regard the wind as "strong" if $v_{\mathrm{w}}>v_{\text {esc }}$ but "weak" if $v_{\mathrm{w}}<v_{\text {esc }}$. The term of "He" or "Fe" wind denotes that the wind is accelerated by the peak of iron lines near $\log T(\mathrm{~K}) \sim 5.2$ or of helium lines near $\log T(\mathrm{~K}) \sim 4.6$. The dashed line indicates the demarcation between the "strong" wind and the "weak" wind.

by using equation (1) of [24]. The realization frequencies of SNe Ia through the $\mathrm{WD}+\mathrm{RG}$ and $\mathrm{WD}+\mathrm{MS}$ channels are estimated as $\sim 0.0017 \mathrm{yr}^{-1}(\mathrm{WD}+\mathrm{RG})$ and $\sim$ $0.001 \mathrm{yr}^{-1}$ (WD+MS), respectively. The total SN Ia rate of the $\mathrm{WD}+\mathrm{MS} / \mathrm{WD}+\mathrm{RG}$ systems becomes $\sim 0.003 \mathrm{yr}^{-1}$, which is close enough to the inferred rate of our Galaxy.

\section{E Low metallicity inhibition of type Ia supernovae}

The optically thick winds are driven by a strong peak of OPAL opacity at $\log T(\mathrm{~K}) \sim 5.2$ (e.g., [32]). Since the opacity peak is due to iron lines, the wind velocity $v_{\mathrm{w}}$ depends on the iron abundance $[\mathrm{Fe} / \mathrm{H}]([35,36])$, i.e., $v_{\mathrm{w}}$ is higher for larger $[\mathrm{Fe} / \mathrm{H}]$. The metallicity effect on SNe Ia is clearly demonstrated by the size of the regions to produce SNe Ia in the diagram of the initial orbital period versus initial mass of the companion star (see Fig. 2). The SN Ia regions are much smaller for lower metallicity because the wind becomes weaker.

The wind velocity depends also on the luminosity $L$ of the WD. The more massive WD has a higher $L$, thus blowing higher velocity winds ( [15]). In order for the wind velocity to exceed the escape velocity of the WD near the photosphere, the WD mass should be larger than a certain critical mass for a given $[\mathrm{Fe} / \mathrm{H}]$. This implies that the initial mass of the WD $M_{\mathrm{WD}, 0}$ should already exceed that critical mass in order for the WD mass to grow to the Ch mass. This critical mass is larger for smaller $[\mathrm{Fe} / \mathrm{H}]$, reaching $1.1 M_{\odot}$ for $[\mathrm{Fe} / \mathrm{H}]=-1.1(\mathrm{Fig}$. 3). Here we should note that the relative number of WDs with $M_{\mathrm{WD}, 0} \gtrsim 1.1 M_{\odot}$ is quite small in close 
binary systems $([47])$. And for $M_{\mathrm{WD}, 0} \gtrsim 1.2 M_{\odot}$, the accretion leads to collapse rather than SNe Ia ( [30]). Therefore, no SN Ia occurs at $[\mathrm{Fe} / \mathrm{H}] \leq-1.1$ in our model.

It is possible to test the metallicity effects on $\mathrm{SNe}$ Ia with the chemical evolution of galaxies.

In the one-zone uniform model for the chemical evolution of the solar neighborhood, the heavy elements in the metal-poor stars originate from the mixture of the SN II ejecta of various progenitor masses. The abundances averaged over the progenitor masses of SNe II predicts $[\mathrm{O} / \mathrm{Fe}] \sim 0.45$ (e.g., $[37,38]$ ). Later $\mathrm{SNe} \mathrm{Ia}$ start ejecting mostly $\mathrm{Fe}$, so that $[\mathrm{O} / \mathrm{Fe}]$ decreases to $\sim 0$ around $[\mathrm{Fe} / \mathrm{H}] \sim 0$. The low-metallicity inhibition of SNe Ia predicts that the decrease in $[\mathrm{O} / \mathrm{Fe}]$ starts at $[\mathrm{Fe} / \mathrm{H}] \sim-1$. Such an evolution of $[\mathrm{O} / \mathrm{Fe}]$ well explains the observations $([35])$.

However, we should note that some anomalous stars have $[\mathrm{O} / \mathrm{Fe}] \sim 0$ at $[\mathrm{Fe} / \mathrm{H}]$ $\lesssim-1$. The presence of such stars, however, is not in conflict with our SNe Ia models, but can be understood as follows: The formation of such anomalous stars (and the diversity of $[\mathrm{O} / \mathrm{Fe}]$ in general) indicates that the interstellar materials were not uniformly mixed but contaminated by only a few SNe II (or even single SN II) ejecta. This is because the timescale of mixing was longer than the time difference between the supernova event and the next generation star formation. The iron and oxygen abundances produced by a single SN II vary depending on the mass, energy, mass cut, and metallicity of the progenitor. Relatively smaller mass SNe II $\left(13-15 M_{\odot}\right)$ and higher explosion energies tend to produce $[\mathrm{O} / \mathrm{Fe}] \sim 0([38,39])$. Those metal poor stars with $[\mathrm{O} / \mathrm{Fe}] \sim 0$ may be born from the interstellar medium polluted by such SNe II.

The metallicity effect on SNe Ia can also be checked with the metallicity of the host galaxies of nearby SNe Ia. There has been no evidence that SNe Ia have occurred in galaxies with a metallicity of $[\mathrm{Fe} / \mathrm{H}] \lesssim-1$, although host galaxies are detected only for one third of SNe Ia and the estimated metallicities of host galaxies are uncertain. Three SNe Ia are observed in low-metallicity dwarf galaxies; SN1895B and SN1972E in NGC 5253, and SN1937C in IC 4182. Metallicities of these galaxies are estimated to be $[\mathrm{O} / \mathrm{H}]=-0.25$ and -0.35 , respectively [40]. If $[\mathrm{O} / \mathrm{Fe}] \sim 0$ as in the Magellanic Clouds, $[\mathrm{Fe} / \mathrm{H}] \sim-0.25$ and -0.35 which are not so small. Even if these galaxies have extremely SN II like abundance as $[\mathrm{O} / \mathrm{Fe}]$ $\sim 0.45,[\mathrm{Fe} / \mathrm{H}] \sim-0.7$ and -0.8 (being higher than -1 ), respectively. Since these host galaxies are blue $(B-V=0.44$ for NGC 5253 and $B-V=0.37$ for IC 4182 according to RC3 catalog), the MS+WD systems are dominant progenitors for the present SNe Ia. The rate of SNe Ia originated from the MS+WD systems is not so sensitive to the metallicity as far as $[\mathrm{Fe} / \mathrm{H}]>-1$ ( $[36])$. Even if $[\mathrm{Fe} / \mathrm{H}] \sim-0.7$ in such blue galaxies, therefore, the SN Ia rate is predicted to be similar to those in more metal-rich galaxies. 


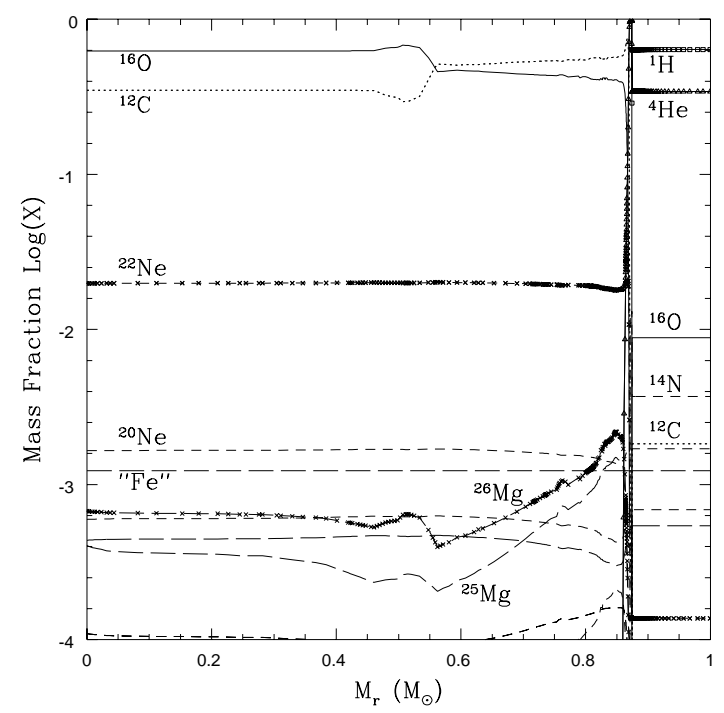

FIGURE 4. Abundances in mass fraction in the inner core of the $6 M_{\odot}$ star for $Y=0.2775$ and $Z=0.02$ at the end of the second dredge-up.

\section{THE ORIGIN OF DIVERSITY OF SNE IA AND ENVIRONMENTAL EFFECTS}

There are some observational indications that SNe Ia are affected by their envi-

ronment. The most luminous SNe Ia seem to occur only in spiral galaxies, while both spiral and elliptical galaxies are hosts for dimmer SNe Ia. Thus the mean peak brightness is dimmer in ellipticals than in spiral galaxies [41]. The SNe Ia rate per unit luminosity at the present epoch is almost twice as high in spirals as in ellipticals [42]. Moreover, [43,44] found that the variation of the peak brightness for $\mathrm{SNe}$ located in the outer regions in galaxies is smaller.

$[45,46]$ examined how the initial composition of the WD (metallicity and the $\mathrm{C} / \mathrm{O}$ ratio) affects the observed properties of $\mathrm{SNe}$ Ia. [47] obtained the $\mathrm{C} / \mathrm{O}$ ratio as a function of the main-sequence mass and metallicity of the WD progenitors. [17] suggested that the variation of the $\mathrm{C} / \mathrm{O}$ ratio is the main cause of the variation of $\mathrm{SNe}$ Ia brightness, with larger $\mathrm{C} / \mathrm{O}$ ratio yielding brighter $\mathrm{SNe}$ Ia. We will show that the $\mathrm{C} / \mathrm{O}$ ratio depends indeed on environmental properties, such as the metallicity and age of the companion of the WD, and that our model can explain most of the observational trends discussed above. We then make some predictions about the brightness of SN Ia at higher redshift.

\section{A $\mathrm{C} / \mathrm{O}$ ratio in $\mathrm{WD}$ progenitors}

In this section we discuss how the $\mathrm{C} / \mathrm{O}$ ratio in the $\mathrm{WD}$ depends on the metallicity and age of the binary system. The $\mathrm{C} / \mathrm{O}$ ratio in $\mathrm{C}+\mathrm{O}$ WDs depends primarily on the main-sequence mass of the WD progenitor and on metallicity. 
We calculated the evolution of intermediate-mass $\left(3-9 M_{\odot}\right)$ stars for metallicity $Z=0.001-0.03$. In the ranges of stellar masses and $Z$ considered in this paper, the most important metallicity effect is that the radiative opacity is smaller for lower $Z$. Therefore, a star with lower $Z$ is brighter, thus having a shorter lifetime than a star with the same mass but higher $Z$. In this sense, the effect of reducing metallicity for these stars is almost equivalent to increasing a stellar mass.

For stars with larger masses and/or smaller $Z$, the luminosity is higher at the same evolutionary phase. With a higher nuclear energy generation rate, these stars have larger convective cores during $\mathrm{H}$ and He burning, thus forming larger He and $\mathrm{C}-\mathrm{O}$ cores.

As seen in Figure 4, the central part of these stars is oxygen-rich. The $\mathrm{C} / \mathrm{O}$ ratio is nearly constant in the innermost region, which was a convective core during $\mathrm{He}$ burning. Outside this homogeneous region, where the $\mathrm{C}-\mathrm{O}$ layer grows due to $\mathrm{He}$ shell burning, the $\mathrm{C} / \mathrm{O}$ ratio increases up to $\mathrm{C} / \mathrm{O} \gtrsim 1$; thus the oxygen-rich core is surrounded by a shell with $\mathrm{C} / \mathrm{O} \gtrsim 1$. In fact this is a generic feature in all models we calculated. The $\mathrm{C} / \mathrm{O}$ ratio in the shell is $\mathrm{C} / \mathrm{O} \simeq 1$ for the star as massive as $\sim 7 M_{\odot}$, and $\mathrm{C} / \mathrm{O}>1$ for less massive stars.

When a progenitor reaches the critical mass for the SNe Ia explosion, the central core is convective up to around $1.1 M_{\odot}$. Hence the relevant $\mathrm{C} / \mathrm{O}$ ratio is between the central value before convective mixing and the total $\mathrm{C} / \mathrm{O}$ of the whole WD. Using the results from the C6 model [19], we assume that the convective region is $1.14 M_{\odot}$ and for simplicity, $\mathrm{C} / \mathrm{O}=1$ outside the $\mathrm{C}-\mathrm{O}$ core at the end of second dredge-up. Then we obtain the $\mathrm{C} / \mathrm{O}$ ratio of the inner part of the $\mathrm{SNe}$ Ia progenitors (Fig. 5).

From this figure we find three interesting trends. First, while the central C/O is a complicated function of stellar mass [47], as shown here the $\mathrm{C} / \mathrm{O}$ ratio in the core before SNe Ia explosion is a decreasing monotonic function of mass. The central $\mathrm{C} / \mathrm{O}$ ratio at the end of second dredge-up decreases with mass for $M_{\mathrm{ms}} \gtrsim 5 M_{\odot}$, while the ratio increases with mass for $M_{\mathrm{ms}} \gtrsim 4 M_{\odot}$; however, the convective core mass during He burning is smaller for a less massive star, and the $\mathrm{C} / \mathrm{O}$ ratio during shell He burning is larger for smaller $\mathrm{C}+\mathrm{O}$ core. Hence, when the $\mathrm{C} / \mathrm{O}$ ratio is averaged over $1.1 M_{\odot}$ the $\mathrm{C} / \mathrm{O}$ ratio decreases with mass. Second, as shown in [47], although the $\mathrm{C} / \mathrm{O}$ ratio is a complicated function of metallicity and mass, the metallicity dependence is remarkably converged when the ratio is seen as a function of the $\mathrm{C}+\mathrm{O}$ core mass $\left(M_{\mathrm{CO}}\right)$ instead of the initial main sequence mass.

According to the evolutionary calculations for $3-9 M_{\odot}$ stars by [47], the $\mathrm{C} / \mathrm{O}$ ratio and its distribution are determined in the following evolutionary stages of the close binary.

(1) At the end of central He burning in the 3-9 $M_{\odot}$ primary star, $\mathrm{C} / \mathrm{O}<1$ in the convective core. The mass of the core is larger for more massive stars.

(2) After central He exhaustion, the outer $\mathrm{C}+\mathrm{O}$ layer grows via He shell burning, where $\mathrm{C} / \mathrm{O} \gtrsim 1[47]$.

(3a) If the primary star becomes a red giant (case $\mathrm{C}$ evolution; e.g., [48]), it then undergoes the second dredge-up, forming a thin He layer, and enters the AGB 


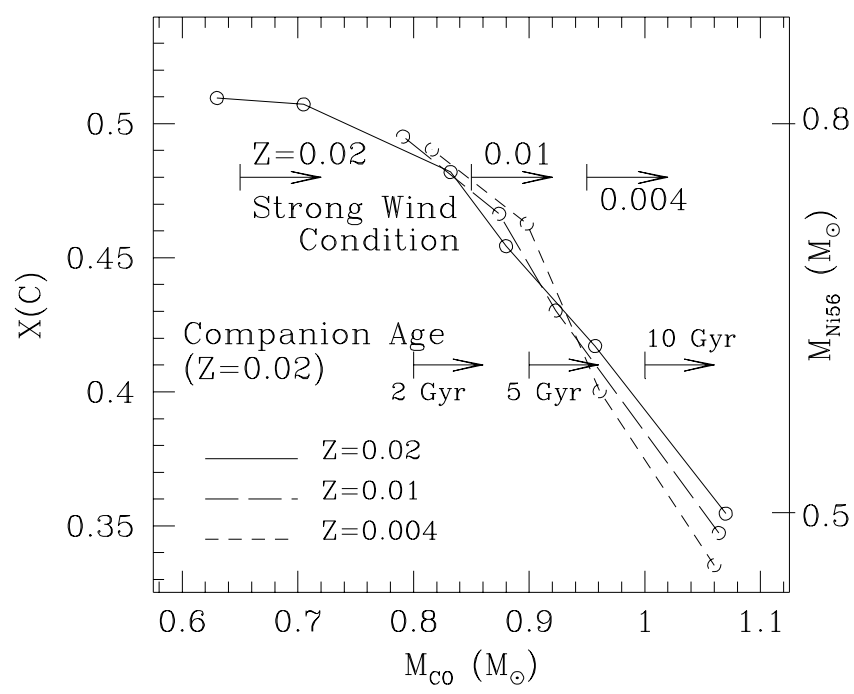

FIGURE 5. The total ${ }^{12} \mathrm{C}$ mass fraction included in the convective core of mass, $M=1.14 M_{\odot}$, just before the $\mathrm{SN}$ Ia explosion as a function of the $\mathrm{C}+\mathrm{O}$ core mass before the onset of mass accretion, $M_{\mathrm{CO}}$. The lower bounds of $M_{\mathrm{CO}}$ obtained from the age effects and the conditions for strong wind to blow are also shown by arrows.

phase. The $\mathrm{C}+\mathrm{O}$ core mass, $M_{\mathrm{CO}}$, at this phase is larger for more massive stars. For a larger $M_{\mathrm{CO}}$ the total carbon mass fraction is smaller.

(3b) When it enters the AGB phase, the star greatly expands and is assumed here to undergo Roche lobe overflow (or a super-wind phase) and to form a $\mathrm{C}+\mathrm{O}$ $\mathrm{WD}$. Thus the initial mass of the WD, $M_{\mathrm{WD}, 0}$, in the close binary at the beginning of mass accretion is approximately equal to $M_{\mathrm{CO}}$.

(4a) If the primary star becomes a He star (case BB evolution), the second dredge-up in (3a) corresponds to the expansion of the He envelope.

(4b) The ensuing Roche lobe overflow again leads to a WD of mass $M_{\mathrm{WD}, 0}=$ $M_{\mathrm{CO}}$.

(5) After the onset of mass accretion, the WD mass grows through steady $\mathrm{H}$ burning and weak He shell flashes, as described in the WD wind model. The composition of the growing $\mathrm{C}+\mathrm{O}$ layer is assumed to be $\mathrm{C} / \mathrm{O}=1$.

(6) The WD grows in mass and ignites carbon when its mass reaches $M_{\mathrm{Ia}}=$ $1.367 M_{\odot}$, as in the model C6 of [19]. Because of strong electron-degeneracy, carbon burning is unstable and grows into a deflagration for a central temperature of $8 \times 10^{8}$ $\mathrm{K}$ and a central density of $1.47 \times 10^{9} \mathrm{~g} \mathrm{~cm}^{-3}$. At this stage, the convective core extends to $M_{r}=1.14 M_{\odot}$ and the material is mixed almost uniformly, as in the C6 model.

In Figure 5, we show the carbon mass fraction $X(\mathrm{C})$ in the convective core of this pre-explosive WD, as a function of metallicity $(Z)$ and initial mass of the WD before the onset of mass accretion, $M_{\mathrm{CO}}$. Figure 5 reveals that: 1$) X(\mathrm{C})$ is smaller for larger $M_{\mathrm{CO}} \simeq M_{\mathrm{WD}, 0}$. 2) The dependence of $X(\mathrm{C})$ on metallicity is small when 

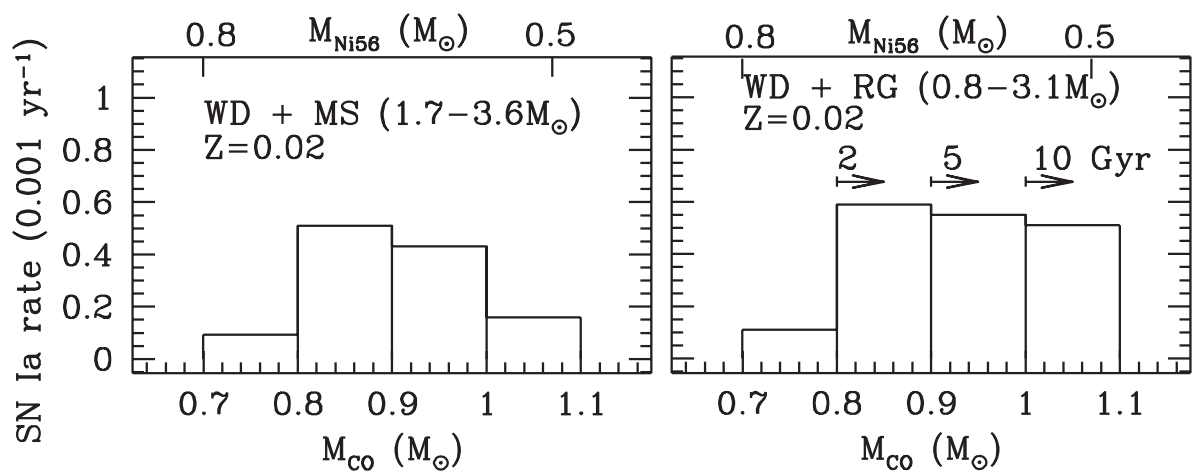

FIGURE 6. SN Ia frequency for a galaxy of mass $2 \times 10^{11} M_{\odot}$ as a function of $M_{\mathrm{CO}}$ for $Z=0.02$. For the WD+RG system, constraints from the companion's age are shown by the arrows. SNe Ia from the WD+MS system occur in spirals but not in ellipticals because of the age effect. $M_{\mathrm{CO}}$ and $M_{\mathrm{Ni56}}$ is assumed to be related as shown here.

plotted against $M_{\mathrm{CO}}$, even though the relation between $M_{\mathrm{CO}}$ and the initial stellar mass depends sensitively on $Z$ [47].

\section{$\mathrm{B}$ Brightness of $\mathrm{SNe} \mathrm{Ia}$ and the $\mathrm{C} / \mathrm{O}$ ratio}

In the Chandrasekhar mass models for SNe Ia, the brightness of SNe Ia is determined mainly by the mass of ${ }^{56} \mathrm{Ni}$ synthesized $\left(M_{\mathrm{Ni} 56}\right)$. Observational data suggest that $M_{\mathrm{Ni56}}$ for most SNe Ia lies in the range $M_{\mathrm{Ni56}} \sim 0.4-0.8 M_{\odot}$ (e.g., [49]). This range of $M_{\mathrm{Ni} 56}$ can result from differences in the $\mathrm{C} / \mathrm{O}$ ratio in the progenitor WD as follows.

In the deflagration model, a larger $\mathrm{C} / \mathrm{O}$ ratio leads to the production of more nuclear energy and buoyancy force, thus leading to a faster propagation. The faster propagation of the convective deflagration wave results in a larger $M_{\mathrm{Ni56}}$. For example, a variation of the propagation speed by $15 \%$ in the W6 - W8 models results in $M_{\mathrm{Ni56}}$ values ranging between 0.5 and $0.7 M_{\odot}$ [19], which could explain the observations.

In the delayed detonation model, $M_{\mathrm{Ni} 56}$ is predominantly determined by the deflagration-to-detonation-transition (DDT) density $\rho_{\mathrm{DDT}}$, at which the initially subsonic deflagration turns into a supersonic detonation [50]. As discussed in [17], $\rho_{\mathrm{DDT}}$ could be very sensitive to $X(\mathrm{C})$, and a larger $X(\mathrm{C})$ is likely to result in a larger $\rho_{\mathrm{DDT}}$ and $M_{\mathrm{Ni} 56}$.

Here we postulate that $M_{\mathrm{Ni} 56}$ and consequently brightness of a SN Ia increase as the progenitors' $\mathrm{C} / \mathrm{O}$ ratio increases (and thus $M_{\mathrm{WD}, 0}$ decreases). As illustrated in Figure 5 , the range of $M_{\mathrm{Ni} 56} \sim 0.5-0.8 M_{\odot}$ is the result of an $X(\mathrm{C})$ range $0.35-0.5$, which is the range of $X(\mathrm{C})$ values of our progenitor models. The $X(\mathrm{C})-M_{\mathrm{Ni} 56}$ - $M_{\mathrm{WD}, 0}$ relation we adopt is still only a working hypothesis, which needs to be proved from studies of the turbulent flame during explosion (e.g., [51]). 


\section{Metallicity and age effects \\ 1 Metallicity effects on the minimum $M_{\mathrm{WD}, 0}$}

As mentioned in $\S 2.5, M_{\mathrm{w}}$ is the metallicity-dependent minimum $M_{\mathrm{WD}, 0}$ for a WD to become an SN Ia (strong wind condition in Fig. 5). The upper bound $M_{\mathrm{WD}, 0} \simeq 1.07 M_{\odot}$ is imposed by the condition that carbon should not ignite and is almost independent of metallicity. As shown in Figure 5 , the range of $M_{\mathrm{CO}} \simeq M_{\mathrm{WD}, 0}$ can be converted into a range of $X(\mathrm{C})$. From this we find the following metallicity dependence for $X(\mathrm{C})$ :

(1) The upper bound of $X(\mathrm{C})$, which is determined by the lower limit on $M_{\mathrm{CO}}$ imposed by the metallicity-dependent conditions for a strong wind, e.g., $X(\mathrm{C})$ $\lesssim 0.51,0.46$ and 0.41 , for $Z=0.02,0.01$, and 0.004 , respectively.

(2) On the other hand, the lower bound, $X(\mathrm{C}) \simeq 0.35-0.33$, does not depend much on $Z$, since it is imposed by the maximum $M_{\mathrm{CO}}$.

(3) Assuming the relation between $M_{\mathrm{Ni} 56}$ and $X(\mathrm{C})$ given in Figure 5, our model predicts the absence of brighter SNe Ia in lower metallicity environment.

\section{Age effects on the minimum $M_{\mathrm{WD}, 0}$}

In our model, the age of the progenitor system also constrains the range of $X(\mathrm{C})$ in SNe Ia. In the SD scenario, the lifetime of the binary system is essentially the main-sequence lifetime of the companion star, which depends on its initial mass $M_{2}$. [14,15] have obtained a constraint on $M_{2}$ by calculating the evolution of accreting WDs for a set of initial masses of the WD $\left(M_{\mathrm{WD}, 0} \simeq M_{\mathrm{CO}}\right)$ and of the companion $\left(M_{2}\right)$, and the initial binary period $\left(P_{0}\right)$. In order for the WD mass to reach $M_{\text {Ia }}$, the donor star should transfer enough material at the appropriate accretion rates. The donors of successful cases are divided into two categories: one is composed of slightly evolved main-sequence stars with $M_{2} \sim 1.7-3.6 M_{\odot}$ (for $Z=0.02$ ), and the other of red-giant stars with $M_{2} \sim 0.8-3.1 M_{\odot}$ (for $Z=0.02$ ) (Fig. 1).

If the progenitor system is older than $2 \mathrm{Gyr}$, it should be a system with a donor star of $M_{2}<1.7 M_{\odot}$ in the red-giant branch. Systems with $M_{2}>1.7 M_{\odot}$ become SNe Ia in a time shorter than 2 Gyr. Likewise, for a given age of the progenitor system, $M_{2}$ must be smaller than a limiting mass. This constraint on $M_{2}$ can be translated into the presence of a minimum $M_{\mathrm{CO}}$ for a given age, as follows: For a smaller $M_{2}$, i.e. for the older system, the total mass which can be transferred from the donor to the WD is smaller. In order for $M_{\mathrm{WD}}$ to reach $M_{\mathrm{Ia}}$, therefore, the initial mass of the $\mathrm{WD}, M_{\mathrm{WD}, 0} \simeq M_{\mathrm{CO}}$, should be larger. This implies that the older system should have larger minimum $M_{\mathrm{CO}}$ as indicated in Figure 5 . Using the $X(\mathrm{C})-M_{\mathrm{CO}}$ and $M_{\mathrm{Ni} 56}-X(\mathrm{C})$ relations (Fig. 5), we conclude that WDs in older progenitor systems have a smaller $X(\mathrm{C})$, and thus produce dimmer SNe Ia.

\section{Comparison with observations}

The first observational indication which can be compared with our model is the possible dependence of the SN brightness on the morphology of the host galaxies. 
[41] found that the most luminous SNe Ia occur in spiral galaxies, while both spiral and elliptical galaxies are hosts to dimmer SNe Ia. Hence, the mean peak brightness is lower in elliptical than in spiral galaxies.

In our model, this property is simply understood as the effect of the different age of the companion. In spiral galaxies, star formation occurs continuously up to the present time. Hence, both $\mathrm{WD}+\mathrm{MS}$ and $\mathrm{WD}+\mathrm{RG}$ systems can produce SNe Ia. In elliptical galaxies, on the other hand, star formation has long ended, typically more than 10 Gyr ago. Hence, WD+MS systems can no longer produce SNe Ia. In Figure 6, we show the frequency of the expected SN I for a galaxy of mass $2 \times 10^{11} M_{\odot}$ for $\mathrm{WD}+\mathrm{MS}$ and $\mathrm{WD}+\mathrm{RG}$ systems separately as a function of $M_{\mathrm{CO}}$. Here we use the results of $[15,14]$, and the $M_{\mathrm{CO}}-X(\mathrm{C})$ and $M_{\mathrm{Ni56}}-X(\mathrm{C})$ relations given in Figure 5. Since a WD with smaller $M_{\mathrm{CO}}$ is assumed to produce a brighter SN Ia (larger $M_{\mathrm{Ni56}}$ ), our model predicts that dimmer SNe Ia occur both in spirals and in ellipticals, while brighter ones occur only in spirals. The mean brightness is smaller for ellipticals and the total SN Ia rate per unit luminosity is larger in spirals than in ellipticals. These properties are consistent with observations.

The second observational suggestion is the radial distribution of SNe Ia in galaxies. $[43,6]$ found that the variation of the peak brightness for SNe Ia located in the outer regions in galaxies is smaller. This behavior can be understood as the effect of metallicity. As shown in Figure 5, even when the progenitor age is the same, the minimum $M_{\mathrm{CO}}$ is larger for a smaller metallicity because of the metallicity dependence of the WD winds. Therefore, our model predicts that the maximum brightness of SNe Ia decreases as metallicity decreases. Since the outer regions of galaxies are thought to have lower metallicities than the inner regions [52,53], our model is consistent with observations. [43] also claimed that SNe Ia may be deficient in the bulges of spiral galaxies. This can be explained by the age effect, because the bulge consists of old population stars.

\section{E Evolution of SNe Ia at high redshift}

We have suggested that $X(\mathrm{C})$ is the quantity very likely to cause the diversity in $M_{\mathrm{Ni} 56}$ and thus in the brightness of SNe Ia. We have then shown that our model predicts that the brightness of SNe Ia depends on the environment, in a way which is qualitatively consistent with the observations. Further studies of the propagation of the turbulent flame and the DDT are necessary in order to actually prove that $X(\mathrm{C})$ is the key parameter.

Our model predicts that when the progenitors belong to an old population, or to a low metal environment, the number of very bright SNe Ia is small, so that the variation in brightness is also smaller, which is shown in Figure 7 . In spiral galaxies, the metallicity is significantly smaller at redshifts $z \gtrsim 1$, and thus both the mean brightness of SNe Ia and its range tend to be smaller (Fig. 7). At $z \gtrsim 2$ SNe Ia would not occur in spirals at all because the metallicity is too low. In elliptical galaxies, on the other hand, the metallicity at redshifts $z \sim 1-3$ is not very different from the present value. However, the age of the galaxies at $z \simeq 1$ is 

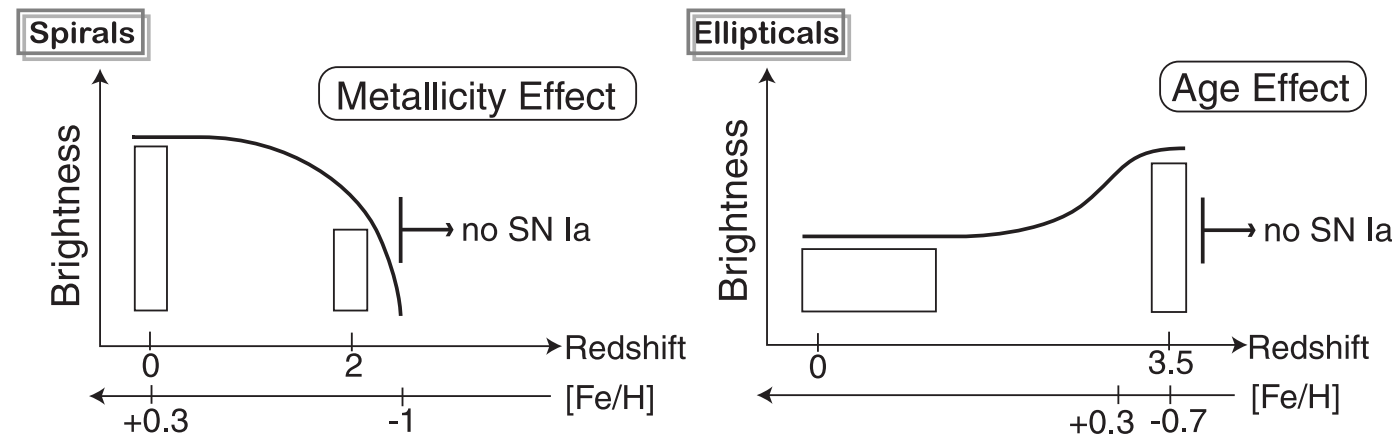

FIGURE 7. Illustration of the predicted variation in SN Ia brightness with redshift.

only about 5 Gyr, so that the mean brightness of SNe Ia and its range tend to be larger at $z \gtrsim 1$ than in the present ellipticals because of the age effect.

We note that the variation of $X(\mathrm{C})$ is larger in metal-rich nearby spirals than in high redshift galaxies. Therefore, if $X(\mathrm{C})$ is the main parameter responsible for the diversity of SNe Ia, and if the LCS method is confirmed by the nearby SNe Ia data, the LCS method can also be used to determine the absolute magnitude of high redshift SNe Ia.

\section{F Possible evolutionary effects}

In the above subsections, we consider the metallicity effects only on the $\mathrm{C} / \mathrm{O}$ ratio; this is just to shift the main-sequence mass $-M_{\mathrm{WD}, 0}$ relation, thus resulting in no important evolutionary effect. However, some other metallicity effects could give rise to evolution of SNe Ia between high and low redshifts (i.e., between low and high metallicities).

Here we point out just one possible metallicity effect on the carbon ignition density in the accreting WD. The ignition density is determined by the competition between the compressional heating due to accretion and the neutrino cooling. The neutrino emission is enhanced by the local Urca shell process of, e.g., ${ }^{21} \mathrm{Ne}^{21} \mathrm{~F}$ pair [54]. (Note that this is different from the convective Urca neutrino process). For higher metallicity, the abundance of ${ }^{21} \mathrm{Ne}$ is larger so that the cooling is larger. This could delay the carbon ignition until a higher central density is reached [55].

Since the WD with a higher central density has a larger binding energy, the kinetic energy of SNe Ia tends to be smaller if the same amount of ${ }^{56} \mathrm{Ni}$ is produced. This might cause a systematically slower light curve evolution at higher metallicity environment. The carbon ignition process including these metallicity effects as well as the convective Urca neutrino process need to be studied (see also [56] for nucleosynthesis constraints on the ignition density).

\section{COSMIC SUPERNOVA RATES}

Attempts have been made to predict the cosmic supernova rates as a function of redshift by using the observed cosmic star formation rate (SFR) [57-59]. The 


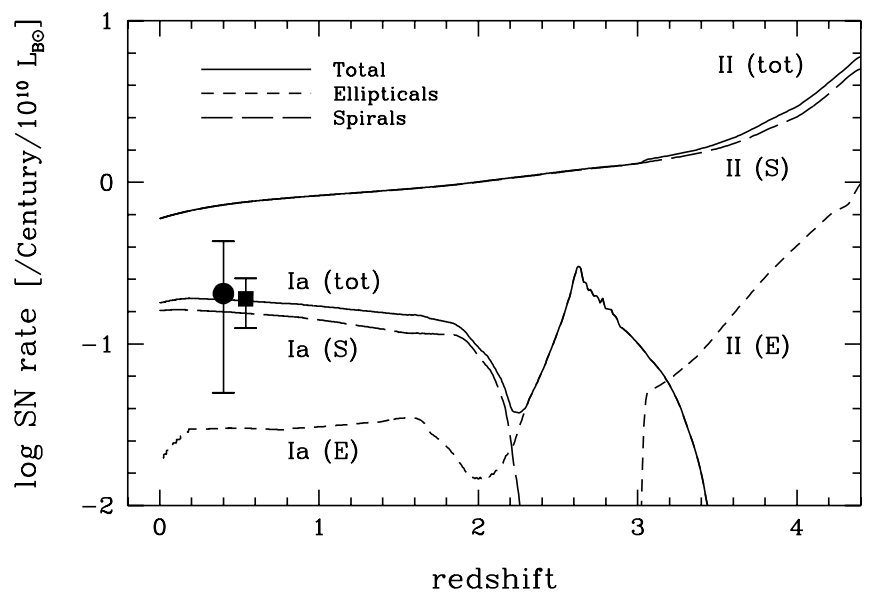

FIGURE 8. The cosmic supernova rates (solid line) as the composite of ellipticals (short-dashed line) and spirals (long-dashed line). The upper three lines show SN II rates, the lower three lines show SN Ia rates. Observational data sources: circle, [63]; square, [10].

observed cosmic SFR shows a peak at $z \sim 1.4$ and a sharp decrease to the present [60]. However, UV luminosities which is converted to the SFRs may be affected by the dust extinction [61]. Recent updates of the cosmic SFR suggest that a peak lies around $z \sim 3$.

[35] predicts that the cosmic SN Ia rate drops at $z \sim 1-2$, due to the metallicitydependence of the SN Ia rate. Their finding that the occurrence of SNe Ia depends on the metallicity of the progenitor systems implies that the SN Ia rate strongly depends on the history of the star formation and metal-enrichment. The universe is composed of different morphological types of galaxies and therefore the cosmic SFR is a sum of the SFRs for different types of galaxies. As each morphological type has a unique star formation history, we should decompose the cosmic SFR into the SFR belonging to each type of galaxy and calculate the SN Ia rate for each type of galaxy.

Here we first construct the detailed evolution models for different type of galaxies which are compatible with the stringent observational constraints, and apply them to reproduce the cosmic SFR for two different environments, e.g., the cluster and the field. Secondly with the self-consistent galaxy models, we calculate the SN rate history for each type of galaxy and predict the cosmic supernova rates as a function of redshift.

\section{A In Clusters}

Galaxies that are responsible for the cosmic SFR have different timescales for the heavy-element enrichment, and the occurrence of supernovae depends on the metallicity therein. Therefore we calculate the cosmic supernova rate by summing up the supernova rates in spirals (S0a-Sa, Sab-Sb, Sbc-Sc, and Scd-Sd) and ellipticals with the ratio of the relative mass contribution. The relative mass contribution is 
obtained from the observed relative luminosity proportion and the calculated mass to light ratio in B-band [18]. The photometric evolution is calculated with the spectral synthesis population database taken from [62]. We adopt $H_{0}=50 \mathrm{~km} \mathrm{~s}^{-1}$ $\mathrm{Mpc}^{-1}, \Omega_{0}=0.2, \lambda_{0}=0$, and the galactic age of $t_{\text {age }}=15 \mathrm{Gyr}$.

First, we make a prediction of the cosmic supernova rates by using the galaxy models which are constructed to meet the observational constraints of cluster galaxies. We assume that elliptical galaxies are formed by a single star burst and stop the star formation at $t \sim 1$ Gyr due to a supernova-driven galactic wind, while spiral galaxies are formed by a relatively continuous star formation. The infall rates and the SFRs are given by [18]. These models are constructed to meet the latest observational constraints such as the present gas fractions and colors for spirals, and the mean stellar metallicity and the color evolution from the present to $z \sim 1$ for ellipticals (see [18] for the figures).

The synthesized cosmic SFR has an excess at $z \gtrsim 3$ due to the early star burst in ellipticals and a shallower slope from the present to the peak at $z \sim 1.4$, compared with Madau's plot [60]. Figure 8 shows the cosmic supernova rates in cluster galaxies. The $\mathrm{SN}$ Ia rate in spirals drops at $z \sim 1.9$ because of the low-metallicity inhibition of SNe Ia. We can test the metallicity effect by finding this drop of the SN Ia in spirals, if high-redshift SNe Ia at $z \gtrsim 1.5$ and their host galaxies are observed with the Next Generation Space Telescope. In ellipticals, the chemical enrichment takes place so early that the metallicity is large enough to produce $\mathrm{SNe}$ Ia at $z \gtrsim 2$. The two peaks of SN Ia rates at $z \sim 2.6$ and $z \sim 1.6$ come from the $\mathrm{MS}+\mathrm{WD}$ and the RG+WD systems, respectively. The SN Ia rate in ellipticals decreases at $z \sim 2.6$, which is determined from the shortest lifetime of SNe Ia of $\sim 0.5$ Gyr. Thus, the total SN Ia rate decrease at the same redshift as ellipticals, i.e., $z \sim 2.6$. (Note, the decrease of the SN Ia rate at $z \sim 1.6$ disappears if we adopt $z_{\mathrm{f}} \sim 3$, because the peak from the MS+WD systems moves to lower redshifts.)

\section{B In Field}

We also predict the cosmic supernova rates assuming that the formation of ellipticals in field took place for over the wide range of redshifts, which is imprinted in the observed spectra of ellipticals in the Hubble Deep Field [64]. The adopted SFRs are the same as the case of cluster galaxies, but for the formation epochs $z_{\mathrm{f}}$ of ellipticals distribute as $\propto \exp \left(-((z-2) / 2)^{2}\right)$ in the range of $0 \leq z \leq 5$,

The synthesized cosmic SFR has a broad peak around $z \sim 3$, which is in good agreement with the recent sub-mm observation [65]. Figure 9 shows the cosmic supernova rates in field galaxies. As in Figure 8, the SN Ia rate in spirals drops at

$z \sim 1.9$. The averaged SN Ia rate in ellipticals decreases at $z \sim 2.2$ as a result of $\sim 0.5$ Gyr delay of the decrease in the SFR at $z \gtrsim 3$. Then, the total SN Ia rate decreases gradually from $z \sim 2$ to $z \sim 3$.

The rate of SNe II in ellipticals evolves following the SFR without time delay. Then, it is possible to observe SNe II in ellipticals around $z \sim 1$. The difference in the SN II and Ia rates between cluster and field ellipticals reflects the difference in the galaxy formation histories in the different environments. 


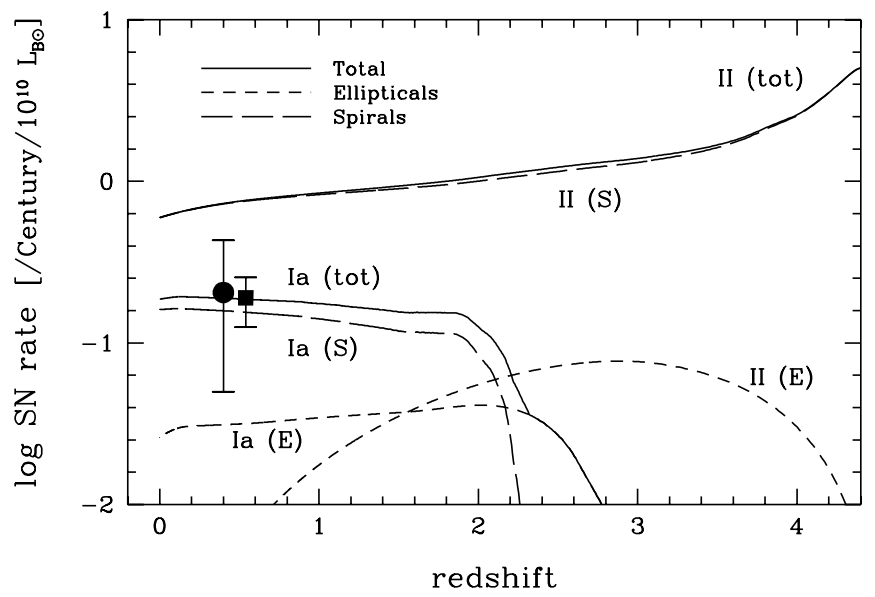

FIGURE 9. The same as Figure 8 , but for the formation epochs of ellipticals span at $1 \lesssim z \lesssim 4$, which is corresponding to field ellipticals.

\section{Summary}

(1) In the cluster environment, the predicted cosmic supernova rate suggests that in ellipticals SNe Ia can be observed even at high redshifts because the chemical enrichment takes place so early that the metallicity is large enough to produce SNe Ia at $z \gtrsim 2.5$. In spirals the SN Ia rate drops at $z \sim 2$ because of the low-metallicity inhibition of SNe Ia.

(2)In the field environment, ellipticals are assumed to form at such a wide range of redshifts as $1 \lesssim z \lesssim 4$. The SN Ia rate is expected to be significantly low beyond $z \gtrsim 2$ because the SN Ia rate drops at $z \sim 2$ in spirals and gradually decreases from $z \sim 2$ in ellipticals.

\section{REFERENCES}

1. Branch, D. 1998, ARA\&A, 1998, 36, 17

2. Gilliland, R. L., Nugent, P. E., \& Phillips, M. M. 1999, ApJ, in press (astro-ph/9903229)

3. Perlmutter, S. et al. 1997, ApJ, 483, 565

4. Perlmutter, S. et al. 1999, ApJ, 517, 565

5. Garnavich, P. et al. 1998, ApJ, 493, 53

6. Riess, A. G. et al. 1998, AJ, 116, 1009

7. Phillips, M. M. 1993, ApJ, 413, L75

8. Hamuy, M., et al. 1995, AJ, 109, 1

9. Riess, A. G., Press, W. H., \& Kirshner, R. P. 1995, ApJ, 438, L17

10. Pain, R. 1999, Talk at the Type Ia supernova workshop in Aspen

11. Nomoto, K., Iwamoto, K., \& Kishimoto, N. 1997a, Science, 276, 1378

12. Branch, D., Livio, M., Yungelson, L. R., Boffi, F. R., \& Baron, E. 1995, PASP, 107, 717

13. Hachisu, I., Kato, M., \& Nomoto, K., 1996, ApJ, 470, L97

14. Hachisu, I., Kato, M., Nomoto, K., \& Umeda, H. 1999a, ApJ, 519, 314

15. Hachisu, I., Kato, M., \& Nomoto, K. 1999b, ApJ, 522, 487

16. Tutukov, A.V., \& Yungelson, L. 1977, Astrophysics, 12, 342

17. Umeda, H., Nomoto, K., Kobayashi, C., Hachisu, I, \& Kato, M. 1999b, ApJ, 522, L43

18. Kobayashi, C., Tsujimoto, T., \& Nomoto, K. 2000, ApJ, submitted (astro-ph/9908005) 
19. Nomoto, K., Thielemann, F. -K., \& Yokoi, K., 1984, ApJ, 286, 644

20. Nomoto, K., Yamaoka, H., Shigeyama, T., Kumagai, S., \& Tsujimoto, T. 1994, in Supernovae, Les Houches Session LIV, ed. S. A. Bludman et al. (North-Holland), 199

21. Arnett, W. D. 1996, Nucleosynthesis and Supernovae (Princeton: Princeton Univ. Press)

22. Höflich, P., \& Khokhlov, A., 1996, ApJ, 457, 500

23. Nugent, P., Baron, E., Branch, D., Fisher, A., \& Hauschildt, P. H. 1997, ApJ, 485, 812

24. Iben, I. Jr., \& Tutukov, A. V. 1984, ApJS, 54, 335

25. Webbink, R. F. 1984, ApJ, 277, 355

26. Nomoto, K. 1982, ApJ, 253, 798

27. Saio, H., \& Nomoto, K. 1985, A\&A, 150, L21

28. Saio, H., \& Nomoto, K. 1998, ApJ, 500, 388

29. Segretain, L., Chabrier, G., \& Mochkovitch, R. 1997, ApJ, 481, 355

30. Nomoto, K., \& Kondo, Y. 1991, ApJ, 367, 119

31. Nomoto, K., Nariai, K., \& Sugimoto, D. 1979, PASJ, 31, 287

32. Iglesias, C. A., \& Rogers, F. 1993, ApJ, 412, 752

33. Kato, M., \& Hachisu, I. 1999, ApJ, 513, L41

34. van den Heuvel, E.P.J., Bhattacharya,D., Nomoto,K., \& Rappaport,S. 1992, A\&A, 262, 97

35. Kobayashi, C., Tsujimoto, T., Nomoto, K., Hachisu, I, \& Kato, M. 1998, ApJ, 503, L155

36. Hachisu, I., \& Kato, M., 2000, in preparation

37. Tsujimoto, T. et al. 1995, MNRAS, 277, 945

38. Nomoto, K. et al. 1997c, Nuclear Physics, A621, 467c

39. Umeda, H., Nomoto, K., \& Nakamura, T. 2000, in The First Stars, ed. A. Weiss et al. (Berlin: Springer), in press (astro-ph/9912248)

40. Kochanek, C. S. 1997, ApJ, 491, 13

41. Hamuy, M., Phillips, M. M., Schommer, R. A., \& Suntzeff, N. B., 1996, AJ, 112, 2391.

42. Cappellaro, E. et al. 1997, A\&A, 322, 431

43. Wang, L., Höflich, P., \& Wheeler, J. C. 1997, ApJ, 483, L29

44. Riess, A. G. et al. 1999, AJ, 117, 707

45. Höflich, P., Wheeler, J. C., \& Thielemann, F. -K., 1998, ApJ, 495, 617

46. Höflich, P., Nomoto, K., Umeda, H., \& Wheeler, J. C., 2000, ApJ, in press

47. Umeda, H., Nomoto, K., Ymamaoka, H., \& Wanajo, S. 1999a, ApJ, 513, 861

48. van den Heuvel, E. P. J., 1994, in Interacting Binaries, eds. S.N. Shore et al. (Berlin: Springer-Verlag), 263

49. Mazzali, P.A., Cappellaro,E., Danziger,I.J., Turatto,M., \& Benetti,S., 1998, ApJ, 499, L49

50. Khokhlov, A. 1991, A\&A, 245, 114

51. Niemeyer J.C., \& Hillebrandt W. 1995, ApJ, 452, 769

52. Zaritsky, D., Kennicutt, R. C., \& Huchra, J. P. 1994, ApJ, 420, 87

53. Kobayashi, C., \& Arimoto, N. 1999, ApJ, 527, 573

54. Paczyński, B. 1973, Acta Astr. 23, 1

55. Nomoto, K. et al. 1997d, in Thermonuclear Supernovae, Eds. P.Ruiz-Lapuente et al. (Dordrecht: Kluwer), 349

56. Iwamoto, K. et al. 1999, ApJS, 125, 439

57. Ruiz-Lapuente, P., \& Canal, R. 1998, ApJ, 497, L57

58. Sadat, R., Blanchard, A., Guiderdoni, B., \& Silk, J. 1998, A\&A, 331, L69

59. Yungelson, L., \& Livio, M. 1998, ApJ, 497, 168

60. Madau, P. et al. 1996, MNRAS, 283, 1388

61. Pettini, M. et al. 1998, ApJ, 508, 539

62. Kodama, T., \& Arimoto, N., 1997, A\&A, 320, 41

63. Pain, R. et al. 1996, ApJ, 473, 356

64. Franceschini, A. et al. 1998, ApJ, 506, 600

65. Hughes, D. et al. 1998, Nature, 394, 241 